\title{
Características individuales y familiares de los adolescentes inmersos en violencia filio-parental: La agresividad física, la cohesión familiar y el conflicto interparental como variables explicativas
}

\author{
Aintzane Zuñeda ${ }^{1}$, Alberto Llamazares ${ }^{1}$, Daniel Marañón ${ }^{2}$ y Gorka Vázquez ${ }^{1}$ \\ ${ }^{1}$ Centro Hobetzen, Asociación Educativa Berriztu, Vizcaya, España \\ ${ }^{2}$ Irrika Centro de Psicología Clínica y Psicoterapia, Vizcaya, España
}

Resumen: El objetivo del presente estudio fue analizar las diferencias en características sociodemográficas y familiares, en salud mental y agresividad de 34 adolescentes inmersos en una dinámica de violencia filio-parental (VFP) y un grupo contraste de 81 adolescentes que no ejercían violencia contra sus progenitores. Los resultados mostraron diferencias entre ambos grupos en variables como nivel de escolarización, estructura familiar, niveles de agresividad física e ira, cohesión, adaptabilidad y comunicación familiar y conflicto interparental, pero no así en los niveles de agresividad verbal y hostilidad, ansiedad, somatización, pánico o depresión. Así mismo los resultados encontrados no apoyaron la hipótesis de que una edad de inicio más temprana de la VFP o una mayor duración de la violencia sin tratamiento se asocien a una mayor gravedad de la VFP. Finalmente, la VFP global se asoció de manera significativa con una mayor agresividad física, una menor cohesión familiar y un alto sentido de autoculpa del adolescente en relación al conflicto interparental.

Palabras clave: Violencia filio-parental; adolescencia; violencia familiar; agresividad.

Characteristics of individuals and families of adolescents in child-to-parent violence: Physical aggressiveness, cohesion within the family and interparental conflict as explanatory variables

\begin{abstract}
The purpose of the present study was to analyse the differences in socio-demographic and family variables, mental health and aggressiveness in a sample of 34 adolescents immersed in dynamics of child-to-parent violence (CPV), and a control group of 81 adolescents who did not abuse their parents. Results showed the existence of differences between the groups in variables such us the level of education, family structure, levels of physical aggression and rage, cohesion, adaptability and communication within the family and interparental conflict, but not as to levels of verbal aggression and hostility, anxiety, somatisation, panic or depression. Moreover, the results did not support the hypothesis that an early start of CPV or a longer duration of violence without treatment are associated with more serious CPV. Finally, global CPV was significantly associated with higher physical aggression, less cohesion within the family and the adolescent's higher autoperception of guilt related to the interparental conflict.
\end{abstract}

Keywords: Child-to-parent violence; adolescence; family violence; aggression.

\section{Introducción}

La violencia Filio-Parental (VFP) puede ser definida como cualquier acto que realiza el menor con la intención

Recibido: 21 enero 2016; aceptado: 23 febrero 2016.

Correspondencia: Alberto Llamazares, Centro Hobetzen, Asociación Educativa Berriztu, C/ Rodriguez Arias 19, 1-d 48008 Bilbao, Vizcaya. Correo-e: resp.pif@berriztu.com de controlar a los padres y/o causarles daño psicológico, físico o financiero (Cottrell, 2001; Cottrell y Monk, 2004).

El aumento de este tipo de violencia durante los últimos años es un hecho constatado a través de los datos

Agradecimientos: Este trabajo se ha realizado gracias a la Asociación Educativa Berriztu, al Servicio de Mujer e Intervención Familiar del Departamento de Inserción Social de la Diputación Foral de Vizcaya y al premio Ayuda a la Investigación 2013 de la Federación Española de Asociaciones de Terapia Familiar (FEATF). 
recogidos en las memorias de la Fiscalía General del Estado del 2006 al 2012. A pesar de ello, no se cuenta con datos fiables entorno a la prevalencia de la misma. Dicha disparidad de datos se debe tal y como afirman Ibabe, Jaureguizar y Bentler (2013a), a la naturaleza secreta del fenómeno así como a la variabilidad de las muestras objeto de estudio, esto es, muestras extraídas de la comunidad que no consultan por una problemática de VFP y muestras procedentes de procesos judiciales iniciados una vez que los progenitores han denunciado a sus hijos.

De acuerdo con el Modelo Ecológico de Cottrell y Monk (2004), es preciso que los factores individuales, interpersonales y contextuales se integren para llevar a cabo una óptima comprensión de la VFP y una posterior intervención. Este modelo asume que es más probable que la VFP ocurra cuando múltiples factores están presentes. De esta manera, las variables que actualmente han tomado fuerza en el estudio de la VFP para su comprensión y estudio pueden ser agrupadas principalmente en un nivel ontogenético y un nivel microsistémico.

En este sentido, en el ámbito ontogenético individual, el abuso de sustancias ha sido identificado como uno de los factores relacionado directamente con el incremento de dicha problemática (Aroca-Montolío, Lorenzo-Moledo y Miró-Pérez, 2014; Calvete, Orue y Sampedro, 2011; Cottrell y Monk, 2004; Evans y Warren-Sohlberg, 1988; Jackson, 2003; Kennedy, Edmonds, Dann y Burnett, 2010; Pagani et al., 2004, 2009). Diversos estudios coinciden en señalar un alto porcentaje de dificultades en el ajuste escolar en aquellos adolescentes que ejercen VFP en comparación con la población general (Nowakowski y Mattern, 2014; Rechea, Fernández y Cuervo, 2008). Respecto al sexo, de manera general se ha informado de una mayor prevalencia entre varones (Aroca-Montolío et al., 2014). Sin embargo, comienzan a hallarse datos que reflejan una prevalencia similar entre hijos e hijas, con la especificidad de que las hijas tienden hacia la utilización de una violencia más psicológica y los hijos más física (Aroca-Montolío et al., 2014; Calvete, Orue y Gámez-Guadix, 2012; Gámez-Guadix y Calvete, 2012; Jaureguizar, Ibabe y Straus, 2013). En relación a la edad, se ha establecido que el rango de mayor incidencia es entre los 10 y 15 años (Aroca-Montolio et al., 2014). Sin embargo, no existen datos respecto a la relación entre la gravedad de la dinámica de la VFP y la edad de los adolescentes ni entre el tiempo de evolución de la problemática y la gravedad y la frecuencia de la misma. Otro de los aspectos estudiados respecto a las características personales y/o ontogenéticas de los adolescentes es la agresividad enfocada desde su dimensión conductual (Ibabe et al., 2013; Kennedy et al., 2010; Walsh y Krienert, 2009). Justamente, se ha informado de un tipo de agresividad proactiva que se relaciona más con un tipo de violencia instrumental que provee de ciertos reforzadores, en lugar de una agresividad reactiva más relacionada con una intensa ira (Calvete y Orue, 2011; Calvete et al., 2012). Sin embargo, ha sido escaso el interés de los estudios sobre la relación entre la VFP y las dimensiones cognitiva y emocional de la agresividad, esto es, la hostilidad y la ira (Berkowitz, 1993). La hostilidad y la ira serían predictores de la conducta agresiva (Arsenio, Cooperman y Lover, 2000) y su estudio orientaría hacia el óptimo tratamiento de la misma. Por otro lado, otro de los factores ontogenéticos estudiados apunta hacia una mayor sintomatología de carácter ansioso-depresivo y una baja autoestima entre los adolescentes que ejercen violencia en el entorno familiar (Aroca-Montolio et al., 2014; Contreras y Cano, 2015; Ibabe y Jaureguizar, 2009; Kennedy et al., 2010). En esta dirección, Calvete et al., (2012) hallaron que la sintomatología depresiva predecía un aumento de la VFP en una amplia muestra de adolescentes procedentes de centros escolares de educación secundaria. Sin embargo, el malestar psicológico y su relación con la VFP no ha sido estudiado en muestras que no constituyan análogos clínicos, esto es, se desconoce cuál es el nivel de malestar psicológico de aquellos adolescentes que requieren de un tratamiento psicológico para tratar la VFP. En este sentido, se hace necesario conocer el malestar psicológico de los adolescentes que acuden a tratamiento para el óptimo desarrollo de tratamientos eficaces.

Respecto al nivel microsistémico, se ha señalado una estrecha relación entre los problemas comportamentales de los niños y determinadas características del contexto y del funcionamiento familiar, como la inadecuada expresión de aceptación y comprensión, la ausencia de cohesión afectiva y de apoyo parental o los problemas de comunicación (Musitu, Estévez y Emler, 2007). Así, en relación al tipo de estructura familiar y a las características de la VFP, resulta complicado extraer de la bibliografía un perfil concreto de familia que esté inmersa en esta. Sin embargo, parece existir un consenso respecto al tipo de composición familiar, avalada por diferentes estudios que hacen referencia a familias en las que la madre es el único progenitor presente en la educación de los adolescentes (Aroca-Montolio et al., 2014; Ibabe, Jaureguizar y Díaz, 2007). Más recientemente, se ha hallado una relación entre el comportamiento agresivo de los adolescentes y la cohesión y adaptabilidad familiar, identificando una correlación negativa entre ellos (Hamama y Arazi, 2011; Ibabe, Jaureguizar y Bentler, 2013b). Sin embargo, en el estudio de la VFP existe una importante laguna a la hora de determinar la posible relación entre el comportamiento agresivo de los hijos hacia sus padres y la percepción que los mismos tienen de la cohesión y adaptabilidad de las familias a las que pertenecen. 
Cuando se hace referencia al funcionamiento familiar desde una perspectiva sistémica, la cohesión y adaptabilidad se consideran dos aspectos centrales en torno a los que giran las interrelaciones de los miembros de la familia. Así, desde el Modelo Circumplejo Familiar (Olson, Sprenkle y Russell, 1979) se recogen dos aspectos bajo la premisa de que los miembros de una familia con un adecuado equilibrio entre cohesión y adaptabilidad mostrarán un mejor desarrollo y adaptación a lo largo del tiempo. La cohesión haría referencia al vínculo emocional entre los diferentes miembros de la familia, mientras la adaptabilidad recogería la capacidad de la familia para cambiar los roles, las reglas relacionales y el liderazgo en respuesta al desarrollo vital o al estrés provocado por diversas situaciones (Olson, y Gorall, 2003).

Asimismo, el conflicto interparental parece ser otro de los aspectos del funcionamiento familiar que puede afectar al desarrollo y a la posterior adaptación de los hijos. El conflicto interparental se entiende como una oposición mutua entre ambos progenitores que refleja la expresión de diferencias entre ambos (Cantón, Cortés y Justicia, 2007). Son numerosos los estudios que establecen una correlación positiva entre la exposición de los hijos al conflicto interparental y posteriores problemas de conducta agresiva y delincuencial en los jóvenes (Cummings, Goeke, Morey y Papp, 2004; El-Sheikh, Buckhalt, Mize y Acebo, 2006). Sin embargo no existen estudios que de manera específica hayan analizado la relación entre el conflicto interparental y la VFP. Por lo tanto, se desconoce la necesidad de un abordaje terapéutico del conflicto interparental en la VFP. Así, el conflicto interparental es entendido como un constructo multidimensional, cuyas dimensiones relevantes en términos de su efecto en el ajuste infantil son la frecuencia, la intensidad, el contenido y la resolución del mismo (Ballesteros de Valderrama, 1995). El impacto que produce el conflicto interparental en los hijos no es directo, sino que depende de cómo se exprese el conflicto y de cómo los hijos interpreten su significado (Iraurgi, Martínez-Pampliega, Iriarte y Sanz, 2011).

De igual manera, diversos estudios apoyan la existencia de una estrecha relación entre el comportamiento violento en la adolescencia y la comunicación negativa entre padres e hijos (Dekovic, Wissink, y Mejier, 2004), así como el papel protector de una óptima comunicación familiar en el desarrollo de un autoconcepto positivo en los hijos (Estévez, Murgui, Moreno y Musitu, 2007). No obstante, se desconoce el papel de la comunicación familiar en la VFP desde la perspectiva de los adolescentes perpetradores de la misma.

De acuerdo con la literatura revisada, existe una notable carencia de estudios empíricos exploratorios con muestras clínicas en VFP que analicen los factores individuales, interpersonales y familiares de manera concurrente para esclarecer el papel diferencial de los mismos en el desarrollo y el mantenimiento de la VFP para así diseñar planes de intervención eficaces. En este sentido, el presente estudio es el único que explora las principales variables asociadas a estos niveles en adolescentes previamente a su inclusión en un tratamiento de VFP (Cottrell y Monk, 2004).

Por ello, el objetivo del presente estudio fue analizar las diferencias existentes entre adolescentes inmersos en una dinámica de VFP y adolescentes que no ejercen violencia familiar en variables decisivas para la implementación de una intervención eficaz, como la sintomatología clínica, la expresión de la agresividad, el funcionamiento y la comunicación familiar, el conflicto interparental y las variables sociodemográficas. Además, se analizó la relación de las variables ontogenéticas y microsistémicas que contribuían a la expresión de la VFP para poder así diseñar óptimos planes de tratamiento psicológico con los adolescentes y sus familias.

Las hipótesis que condujeron el estudio fueron las siguientes: 1) Los adolescentes pertenecientes al grupo VFP mostrarían un nivel de escolarización significativamente inferior al correspondiente por edad, así como una mayor pertenencia a estructuras familiares diferentes de la nuclear en comparación con el grupo contraste (GC), 2) El grupo de adolescentes con VFP mostraría mayores niveles de ansiedad, somatización y pánico, así como mayores niveles de agresividad física y verbal, ira y hostilidad en comparación con los adolescentes del GC, no esperando diferencias significativas en depresión entre ambos grupos, 3) los adolescentes pertenecientes al grupo VFP mostrarían menor cohesión y menor adaptación familiar, así como mayores niveles de conflicto interparental y una deteriorada comunicación familiar en comparación con el GC, 4) Una edad de inicio más temprana de la VFP y una duración mayor de la VFP sin tratamiento, junto a variables relacionadas con el funcionamiento familiar, como una baja cohesión y adaptabilidad y un alto conflicto interparental percibido, predecirían la VFP en el seno familiar.

\section{Método}

\section{Participantes}

Para obtener la muestra del estudio se empleó un muestreo intencional ya que tomando en consideración los objetivos del estudio, no se buscaban resultados generalizables a toda la población adolescente, sino obtener la muestra más representativa posible de adolescentes perpetradores de VFP y que son objeto de 
tratamiento junto a sus familias. Igualmente, la selección del GC tuvo como criterio equiparar la edad y el sexo a la muestra clínica y no cumplir criterios de VFP. El $96.5 \%$ de la muestra global provenía de un entorno urbano y el 100\% eran de nacionalidad española.

Así, en la investigación participaron 34 adolescentes de edades comprendidas entre 10 y 21 años $(M=15.61$, $\mathrm{DT}=2.13$ ) perpetradores de violencia hacia sus padres, de los cuales $47.1 \%$ fueron varones y $52.9 \%$ fueron mujeres. Todos ellos fueron incluidos en un programa de intervención familiar especializado en VFP. La inclusión de los adolescentes en el estudio estuvo supeditada a su pertenencia a familias que habían consultado por una dinámica de VFP. Se excluyeron aquellos adolescentes que, perteneciendo a familias envueltas en una dinámica de violencia intrafamiliar, no se adecuaban a los criterios que definen una casuística de VFP, así como los menores de 10 años y los mayores de 21 . El 23.5\% de los adolescentes en el grupo VFP había sido denunciado por su progenitores, 4 estaban diagnosticados de Trastorno Disocial y 2 de Trastorno por Déficit de Atención e Hiperactividad.

El grupo contraste (GC), conformado por 81 adolescentes que no ejercían VFP en el seno familiar, fue equiparado con el grupo clínico en sexo y edad. Los 81 adolescentes de edades comprendidas entre 13 y 18 años (M $=15.22, \mathrm{DT}=1.53)$, no mantenían el mismo rango de edad que el grupo clínico objeto de estudio debido a que únicamente fue evaluado un participante de 10 años y otro de 21 años en el grupo VFP. El GC provenía de 3 institutos y colegios de educación secundaria públicos y concertados, así como de una asociación educativa de apoyo escolar. Los criterios para incluir a los adolescentes en el GC fueron que perteneciesen a familias que hubiesen mostrado su acuerdo de participación mediante la firma del consentimiento informado y en las que no existiese una problemática de VFP, evaluada mediante la respuesta negativa a cada uno de los ítems del CTS-CP.

\section{Instrumentos y variables}

Variables clínicas y sociodemográficas. En esta parte se incluyó la edad, el sexo, la escolarización, estructura familiar (nuclear-tradicional, padres separados, reconstituida o monoparental), tipo de VFP (psicológica o física y psicológica) recogida mediante entrevista clínica con el sistema familiar, violencia intrafamiliar (violencia marital y/o violencia parento-filial), consumo de tóxicos, edad de inicio de la VFP y tiempo transcurrido en meses hasta la petición de tratamiento.

Escala de Tácticas para Conflictos - Hijo Padres (Conflict Tactics Scales - Child Parents; CTS-CP, Straus y Fauchier, 2008). El CTS-CP consiste en 6 ítems crea- dos en el contexto del International Parenting Study (Straus y Fauchier, 2008) para evaluar actos agresivos de hijos a progenitores y obtenidos a partir del CTS-PC (Straus, Hamby, Finkelhor, Moore, y Runyan, 1998). Tres de los ítems reflejan actos de violencia verbal como insultar, gritar y amenazar con pegar a los progenitores, y tres reflejan actos de violencia física como abofetear, golpear con un objeto que puede causar daño y dar patadas. Los adolescentes contestaron empleando una escala con los siguientes valores: 0 nunca, 1 a veces y 2 a menudo. En la traducción elaborada por Calvete el al. (2011) obtuvieron un coeficiente alpha para la escala completa de $.69, .61$ y .88 para las subescalas de agresiones verbales y agresiones físicas, respectivamente. En el presente estudio, el coeficiente a de consistencia interna para la escala global fue .81, para la subescala de agresiones verbales contra los progenitores fue $.66 \mathrm{y}$ finalmente para la subescala de violencia física fue .6.

El Cuestionario de Agresividad (Agression Questionnaire; AQ) de Buss y Perry (1992) fue utilizado para el estudio de la conducta agresiva en los adolescentes mediante la valoración de la agresión física y la verbal, la hostilidad y la ira, valorando así los aspectos conductuales, cognitivos y emocionales de la agresividad. Para este fin, fue utilizada la versión española reducida del cuestionario de Vigil-Colet, Lorenzo-Seva, CodorniuRaga, y Morales (2005), de 20 ítems en los que se ha de responder en una escala tipo likert desde 1 Completamente falso para mí y 5 Completamente verdadero para mí. Dicha escala ha sido utilizada debido a su ajuste aceptable al modelo de cuatro factores y su adecuada consistencia interna (Morales-Vives, Codorniu-Raga y Vigil-Colet, 2005). El nivel de consistencia interna informado por los autores originales fue satisfactorio para las cuatro escalas; .87 para la escala global, .88 para la agresividad física, .71 para la agresividad verbal y .68 para la ira y .65 para la hostilidad. Igualmente, los coeficientes de fiabilidad encontrados en la presente investigación fueron satisfactorios, esto es, .85 para la escala global, .89 para la agresividad física, .69 para la agresividad verbal y .7 para la ira y la hostilidad.

Brief Symptoms Inventory-18 (BSI-18; Derogatis, 2001), es un breve inventario que tiene por objetivo valorar el distrés psicológico mediante 18 ítems tipo likert de 0 a 4, siendo 0 nada y 4 mucho, existiendo la posibilidad de marcar no contesta. En este sentido, aporta una medida de distrés general y cuatro subescalas: somatización, depresión, ansiedad general y pánico. Andreu et al. (2008) en el examen realizado sobre la consistencia interna de la prueba con una muestra española hallaron un .89 para la dimensión global, .78 para la somatización, .88 para la depresión, .71 para la ansiedad y .78 para el 
pánico. En el presente estudio el a de Cronbach para cada una de las dimensiones fueron .91 para la dimensión global del BSI, .81 para la somatización, .86 para la depresión, .67 para la ansiedad y .72 para el pánico.

Family Adaptability and Cohesion Evaluation Scale (FACES II, Olson, Bell y Portner, 1982) proporciona una medida de la cohesión y la adaptabilidad familiar. La versión breve utilizada para la presente investigación cuenta con 20 ítems que se puntúan mediante una escala de tipo likert siendo 1 nunca o casi nunca y 5 casi siempre (Martínez-Pampliega, Iraurgi, Galíndez y Sanz, 2006). La fiabilidad de ambas subescalas obtenidas por los autores fue de .89 para la cohesión y .87 para la adaptabilidad. En la presente investigación fue comprobada la fiabilidad de ambas subescalas, obteníéndose así una a de Cronbach de .93 para la cohesión y un .92 para la adaptabilidad.

Escala de conflicto interparental desde la perspectiva de los hijos (The Children's perception of interparental conflict scale; CPIC-VER, Grych, Seid y Fincham, 1992). Fue aplicada para estudiar el conflicto interparental desde la percepción de los hijos. La versión española utilizada en el presente estudio (Iraurgi et al., 2008) consta de 36 ítems que se responden con tres opciones de respuesta: 1 verdadero, 2 casi verdadero y 3 falso. Su puntuación tiene la especificidad de que a menor puntuación mayor conflicto interparental percibido por los hijos. El CPICVER comprende las siguientes subescalas para las cuales hallaron los siguientes coeficientes a de consistencia interna: amenaza .81 , autoculpa. 76 , contenido .78 , eficacia .59 , estabilidad .74 , frecuencia .82 , intensidad .77 , resolución .81 y triangulación .5. En el presente estudio se hallaron los siguientes coeficientes a de consistencia interna: amenaza .77 , autoculpa .71 , contenido .79 , eficacia .67 , estabilidad .81 , frecuencia .4 , intensidad .74, resolución .82 y triangulación .60. Las nueve subescalas fueron agrupadas en tres grandes dimensiones: 1) las propiedades del conflicto, con un a de Cronbach de .8 y compuesta por las dimensiones de intensidad, frecuencia, estabilidad y resolución, 2) la vivencia o evaluación amenazante, con un a igual a .79 y compuesta por la eficacia de afrontamiento, la amenaza percibida y la triangulación, y 3 ) la culpabilidad, con un a de Cronbach de .85 y compuesta por las dimensiones de contenido y autoculpa. A nivel global la escala mostró un alpha de Cronbach de .91.

Escala de Comunicación Familiar (Family Communication Scale; FCS-VE) de Barnes y Olson (1982). El presente instrumento es utilizado para evaluar la comunicación en el seno de la familia. La versión utilizada fue la adaptación llevada a cabo por Martínez-Pampliega (2008) de 10 ítems tipo likert en donde puede responderse desde si la afirmación no describe nada mi familia (1) hasta describe muy bien a mí familia (5). El coeficiente a de consistencia interna hallada por Martinez-Pampliega (2008) fue .88, en el presente estudio fue de .91.

\section{Procedimiento}

Para la inclusión de los adolescentes en el presente estudio, primeramente se llevaron a cabo dos entrevistas familiares exploratorias para dilucidar la dinámica de VFP en el seno familiar y la adecuación al programa de tratamiento. Estas entrevistas las llevó a cabo el equipo técnico compuesto por dos psicólogos expertos en la casuística. A continuación, se solicitó a las familias, y a los adolescentes la firma de un consentimiento informado para su participación en el estudio y en el tratamiento familiar. Los adolescentes del grupo clínico cumplimentaron la batería de cuestionarios antes de su inclusión en el tratamiento psicoterapéutico y socioeducativo familiar. Los adolescentes del GC seleccionados entre los cursos $1^{\circ}$ de la ESO y $2^{\circ}$ de Bachillerato, fueron evaluados mediante una aplicación grupal de los cuestionarios en una de las aulas del centro, tras recibir su consentimiento y el de sus padres. Se aseguró el carácter anónimo y confidencial de sus respuestas, a la vez que se les instruyó en la manera de contestar a las pruebas que encontrarían en la batería de cuestionarios.

\section{Análisis de datos}

En un primer momento se analizó la normalidad de la distribución de cada una de las variables estudiadas. Aquellas variables que no siguieron una distribución normal se transformaron mediante las indicaciones propuestas por Field (2005). Para comprobar la no existencia de diferencias significativas en edad y sexo entre las muestras objeto de estudio, fueron utilizadas la prueba $t$-student para muestras independientes y la prueba de $\chi^{2}$ para las variables categóricas. Las diferencias entre VFP y $\mathrm{GC}$ en las variables estudiadas fueron valoradas mediante el análisis de varianza (ANOVA). Para la obtención de una medida objetiva de los tamaños del efecto, la Eta fue analizada tomando como referencia los criterios sugeridos por Cohen (1992). Por otro lado, con objeto de analizar la relación entre las variables en las que se habían obtenido diferencias entre ambos grupos y la VFP, fue calculado el coeficiente de correlación de Pearson. Finalmente, solo las variables que correlacionaron de manera significativa con la VFP en al menos un nivel de significación estadística de $p<.05$, fueron incluidas en el posterior análisis de regresión lineal múltiple como variables predictoras para la variable criterio VFP global, verbal y física. Todos los datos fueron analizados mediante el programa estadístico SPSS 15.0. 
Tabla 1. Características de clínicas y sociodemográficas en el grupo de VFP y GC

\begin{tabular}{|c|c|c|c|c|c|}
\hline & \multicolumn{2}{|c|}{$\operatorname{VFP}(n=34)$} & \multicolumn{2}{|c|}{$\mathrm{GC}(n=81)$} & \multirow[b]{2}{*}{$\chi^{2}$} \\
\hline & $n$ & $\%$ & $n$ & $\%$ & \\
\hline \multicolumn{6}{|l|}{ Sexo } \\
\hline Masculino & 16 & 47.1 & 38 & 46,9 & 0.00 \\
\hline \multicolumn{6}{|l|}{ Escolarización } \\
\hline Nivel correspondiente a edad & 9 & 26.5 & 72 & 90 & $47.74 * * *$ \\
\hline Nivel por debajo a edad & 22 & 64.7 & 8 & 10 & \\
\hline No escolarizado & 3 & 8.8 & 0 & 0 & \\
\hline \multicolumn{6}{|l|}{ Estructura Familiar } \\
\hline Nuclear & 14 & 41.2 & 61 & 75.3 & $16.74^{* * *}$ \\
\hline Padres separados & 15 & 44.1 & 16 & 19.8 & \\
\hline Reconstituida & 3 & 8.8 & 0 & 0 & \\
\hline Monomarental & 2 & 5.9 & 4 & 4.9 & \\
\hline \multicolumn{6}{|l|}{ VFP } \\
\hline Violencia Psicológica & 7 & 20.6 & & & \\
\hline Violencia Física y Psicológica & 27 & 79.4 & & & \\
\hline \multicolumn{6}{|l|}{ Violencia Intrafamiliar } \\
\hline Violencia Marital & 11 & 32.4 & & & \\
\hline Violencia Parento-filial & 3 & 8.8 & & & \\
\hline \multicolumn{6}{|l|}{ Consumo de Tóxicos } \\
\hline Ausencia de consumo & 13 & 38.2 & & & \\
\hline Cannabis & 2 & 5.9 & & & \\
\hline Alcohol & 1 & 2.9 & & & \\
\hline \multirow[t]{2}{*}{ Cannabis y Alcohol } & 18 & 52.9 & & & \\
\hline & \multicolumn{2}{|c|}{ Media $(D T)$} & \multicolumn{2}{|c|}{ Media $(D T)$} & $t$ \\
\hline Edad & \multicolumn{2}{|c|}{$15.61(2.13)$} & \multicolumn{2}{|c|}{$15.22(1.53)$} & 1.12 \\
\hline Edad Inicio VFP & \multicolumn{2}{|c|}{$13.9(1.81)$} & & & \\
\hline Tiempo de Evolución VFP (meses) & \multicolumn{2}{|c|}{$21(9.36)$} & & & \\
\hline \multicolumn{6}{|l|}{ CTS } \\
\hline CTS Violencia Física & \multicolumn{2}{|c|}{$3.97(1.17)$} & \multicolumn{2}{|c|}{$3(0)$} & $4.88^{* * *}$ \\
\hline CTS Violencia Verbal & \multicolumn{2}{|c|}{$6.79(1.5)$} & \multicolumn{2}{|c|}{$4.41(0.94)$} & $8.64^{* * *}$ \\
\hline CTS VFP Global & \multicolumn{2}{|c|}{$10.76(2.21)$} & \multicolumn{2}{|c|}{$7.41(0.94)$} & $8.62^{* * *}$ \\
\hline
\end{tabular}

Nota . CTS $=$ Conflict Tactics Scales; $D T=$ Desviación Típica; $\mathrm{GC}=$ grupo control; VFP = violencia filio-parental.

$* p<.05 ; * * p<.01 ; * * * p<.001$.

\section{Resultados}

En la Tabla 1 pueden observarse el resto de variables clínicas y sociodemográficas de relevancia, así como comprobar la ausencia de diferencias significativas en sexo y edad entre ambos grupos. Respecto al nivel de escolarización y al tipo de estructura familiar, se obtuvieron diferencias significativas.
Mediante el análisis de varianza pudo observarse cómo el grupo VFP mostró diferencias significativas al compararlo con el GC en el conjunto de variables concernientes al sistema familiar a excepción de la variable amenaza perteneciente a la escala CPICS-VER. Los tamaños del efecto obtenidos fueron moderados (Cohen, 1992) (véase Tabla 2). En el mismo sentido, se halló un mayor conflicto interparental significativo en VFP a tra- 
vés de los tres factores pertenecientes a la escala CPICSVER, Propiedades del Conflicto F $(1,113)=26.05 \mathrm{p}<$ $.0001, \eta^{2}=0.19$, Vivencia o Evaluación Amenazante $\mathrm{F}$ $(1,113)=5.8 \mathrm{p}<.018, \eta^{2}=0.05$ y Autoculpabilidad F $(1,113)=33.47 \mathrm{p}<.0001, \eta^{2}=0.23$. Respecto a la com- paración de ambos grupos en las variables clínicas examinadas, no se encontraron diferencias significativas. Sin embargo, en lo concerniente a la agresividad, los adolescentes que expresaban VFP mostraron una mayor agresividad global, física e ira (véase Tabla 3).

Tabla 2. Comparación entre el grupo de adolescentes con VFP y GC en las variables familiares

\begin{tabular}{|c|c|c|c|c|}
\hline & $\operatorname{VFP}(n=34)$ & $\mathrm{GC}(n=81)$ & & \\
\hline Variables Familiares & Media $(D T)$ & Media $(D T)$ & $F(1,13)$ & $\eta^{2}$ \\
\hline \multicolumn{5}{|l|}{ FACES } \\
\hline Cohesión & $26.76(8.13)$ & $38.02(7.24)$ & $53.76 * * *$ & .32 \\
\hline Adaptabilidad & $24.34(8.05)$ & $34.14(7.70)$ & $37.72 * * *$ & .25 \\
\hline \multicolumn{5}{|l|}{ CPICS-VER } \\
\hline Amenaza & $9.6(2.32)$ & $9.82(2.32)$ & 0.22 & 0 \\
\hline Autoculpa & $9.35(1.86)$ & $11.24(1.26)$ & $39.8 * * *$ & .26 \\
\hline Contenido & $8.91(2.04)$ & $10.51(1.67)$ & $20.2 * * *$ & .15 \\
\hline Eficacia & $7.9(2.01)$ & $9.19(2.09)$ & $9.18 * *$ & .07 \\
\hline Estabilidad & $9.16(2.47)$ & $10.73(1.87)$ & $13.87 * * *$ & .11 \\
\hline Frecuencia & $8.26(2.53)$ & $10.8(3.57)$ & $14.21 * * *$ & .11 \\
\hline Intensidad & $6.47(2.04)$ & $7.92(2.19)$ & $11 * * *$ & .9 \\
\hline Resolución & $7.68(2.01)$ & $9.84(2.03)$ & $27.25 * * *$ & .19 \\
\hline Triangulación & $9.68(1.85)$ & $10.72(1.66)$ & $8.76^{* *}$ & .07 \\
\hline \multicolumn{5}{|l|}{ FCS-VE } \\
\hline Comunicación Familiar & $27.73(7.23)$ & $37.49(7.45)$ & $41.73 * * *$ & .27 \\
\hline
\end{tabular}

Notas. CPIC-VER = The Children's Perception of Interparental Conflict Scale-Versión Española Reducida (Iraurgi et al., 2008); FACES = Family Adaptability and Cohesion Evaluation Scale (Olson. Bell y Portner. 1982); FCS-VE = Family Comunication Scale (Barnes y Olson. 1982); GC = grupo control; VFP = violencia filio-parental; ${ }^{*} p<.05 ; * * p<.01 ; * * *<.001$

Tabla 3. Comparación entre el grupo VFP y GC en las variables clínicas y agresividad

\begin{tabular}{|c|c|c|c|c|}
\hline & $\operatorname{VFP}(n=34)$ & $\mathrm{GC}(\mathrm{n}=81)$ & & \\
\hline Variables clínicas y agresividad & Media $(D T)$ & Media $(D T)$ & $F(1,113)$ & $\eta^{2}$ \\
\hline \multicolumn{5}{|l|}{ BSI } \\
\hline BSI Global & $15.48(15.24)$ & $13.45(10.25)$ & 0.76 & 0 \\
\hline Somatización & $3.32(4.62)$ & $3.35(3.99)$ & 0.01 & 0 \\
\hline Depresión & $6.12(6.33)$ & $4.68(4.32)$ & 1.98 & 0 \\
\hline Ansiedad & $4.44(3.42)$ & $3.7(2.67)$ & 1.56 & 0 \\
\hline Pánico & $1.7(2.65)$ & $1.69(2.02)$ & 0 & 0 \\
\hline \multicolumn{5}{|l|}{ AQ } \\
\hline AQ Global & $57.67(14.09)$ & $48.08(10.36)$ & $16.4 * * *$ & .13 \\
\hline Agresividad Física & $21.37(7.02)$ & $14.3(5.55)$ & $33.14 * * *$ & .23 \\
\hline Agresividad Verbal & $10.21(3.75)$ & $10.67(2.97)$ & 0.48 & 0 \\
\hline Ira & $13.96(3.41)$ & $11.37(3.49)$ & $13.37 * * *$ & .11 \\
\hline Hostilidad & $12.12(5.06)$ & $11.78(3.31)$ & 0.21 & 0 \\
\hline
\end{tabular}

Notas. AQ = Agression Questionnaire (Buss y Perry. 1992); BSI = Brief Symptoms Inventory (Derogatis, 2001); GC = grupo control; VFP = violencia filio-parental. ${ }^{*} p<.05 ; * *<.01 ; * * * p<.001$ 
Para evaluar la relación entre las variables en las que se habían obtenido diferencias significativas con la VFP, fue calculado el coeficiente de correlación de Pearson. Así, la VFP no correlacionó de forma significativa con la edad, ni con la edad de inicio de la VFP, ni con la duración de la VFP en el sistema familiar. Sin embargo, la VFP global mantuvo una correlación significativa con la cohesión y adaptabilidad familiar, la autoculpa del adolescente respecto al conflicto interparental y la agresividad física y la ira. La VFP física correlacionó de manera significativa con la cohesión y la adaptabilidad familiar, la autoculpa del adolescente en el conflicto interparental y la comunicación familiar. Se obtuvieron correlaciones significativas entre la VFP verbal y la autoculpa, así como con la agresividad fisica y la ira (véase Tabla 4).

Tabla 4. Coeficientes de correlación entre la VFP y sus variables clínicas y las variables ontogenéticas y microsistémicas

\begin{tabular}{|c|c|c|c|c|c|c|c|}
\hline & \multirow{2}{*}{ Variables } & \multicolumn{3}{|c|}{ CTS VFP } & \multirow[b]{2}{*}{ Edad } & \multirow[b]{2}{*}{ Edad Inicio } & \multirow[b]{2}{*}{ Duración } \\
\hline & & VFP Global & VFP Física & VFP Verbal & & & \\
\hline \multirow[t]{3}{*}{ CTS VFP } & VFP Global & - & $.77 * *$ & $.87 * *$ & .14 & .07 & .24 \\
\hline & VFP Física & $.77 * *$ & - & $.36^{*}$ & -.11 & -.14 & .03 \\
\hline & VFP Verbal & $.87 * *$ & $.36^{*}$ & - & .29 & .21 & .33 \\
\hline \multirow[t]{2}{*}{ FACES } & Cohesión & $-.42 *$ & $-.48 * *$ & -.25 & -.05 & .04 & -.03 \\
\hline & Adaptabilidad & $-.44 * *$ & $-.47 * *$ & -.28 & -.16 & .15 & -.09 \\
\hline \multirow[t]{3}{*}{ CPICS-VER } & Conflicto & -.25 & -.29 & -.14 & .08 & .08 & .03 \\
\hline & Autoculpa & $-.55^{* *}$ & $-.43^{*}$ & $-.46^{* *}$ & -.07 & -.03 & -.12 \\
\hline & Amenaza & -.25 & -.33 & -.11 & .8 & .03 & .17 \\
\hline FCS-VE & Comunicación Familiar & -.29 & $-.36^{*}$ & -.15 & -.05 & 0 & -.14 \\
\hline \multirow[t]{2}{*}{ AQ } & Agresividad Física & $.58 * *$ & .29 & $.62 * *$ & .16 & .07 & .28 \\
\hline & Ira & $.45^{* *}$ & .17 & $.52 * *$ & .11 & .09 & .12 \\
\hline
\end{tabular}

Notas. AQ = Agression Questionnaire (Buss y Perry. 1992); CPIC-VER = The Children's Perception of Interparental Conflict Scale-Versión Española Reducida (Iraurgi et al., 2008); CTS = Conflict Tactics Scales (Straus y Fauchier. 2008); FACES = Family Adaptability and Cohesion Evaluation Scale (Olson. Bell y Portner. 1982); FCS-VE = Family Comunication Scale (Barnes y Olson. 1982). VFP = violencia filio-parental; ${ }^{*} p<.05 ; * * p<.01$

Finalmente, para valorar la influencia de las diferentes variables que correlacionaron de forma significativa en el desarrollo de la VFP tanto global, como física y verbal, se realizó un análisis de regresión lineal múltiple. En este sentido, se introdujeron como variables predictoras la cohesión y la adaptabilidad familiar, la autoculpa, la agresividad física y la ira, y como variable criterio se mantuvo la VFP global. Los resultados indicaron que el modelo final, constituido por la agresividad física, una baja cohesión familiar y un alto sentido de autoculpa por parte del adolescente en relación al conflicto interparental, explicaba el 55\% de la varianza en la VFP global. Sin embargo, únicamente la baja cohesión familiar junto a la autoculpa en el conflicto interparental fueron las variables predictoras que contribuyeron en un $40 \%$ a la varianza de la VFP física. Finalmente, la agresividad física fue la única variable que se constituyó como variable predictora de la varianza de la VFP verbal, explicando el 39\% de la misma (véase Tabla 5).

\section{Discusión}

Los resultados del presente estudio apoyan la primera hipótesis planteada respecto a la existencia de diferencias significativas entre el GC y el de VFP en la proporción de adolescentes que se encuentran escolarizados en un nivel diferente al que le correspondería por edad. Así, se confirman los resultados encontrados en investigaciones españolas previas, en las que los porcentajes de adolescentes con VFP que presentan dificultades académicas variaba entre el 93\% (Ibabe et al., 2007) y el 53\% (Rechea et al, 2008). Las dificultades de estos adolescentes para responder de una manera adaptativa a las exigencias del ámbito académico podrían vincularse, por un lado, con sus dificultades emocionales y déficits en estrategias para solventar problemas interpersonales, $\mathrm{y}$, por otro, con posibles dificultades en la inteligencia académica y/o cognición impersonal, al igual que aquellos adolescentes que muestran conductas antisociales (Aroca-Montolio et al., 2014; Henggeler, 1989). Ade- 
Tabla 5. Análisis de regresión lineal múltiple para la VFP

\begin{tabular}{|c|c|c|c|c|}
\hline \multirow[b]{2}{*}{ Variable criterio } & \multirow[b]{2}{*}{ Modelo } & \multicolumn{3}{|c|}{ Coeficientes } \\
\hline & & $\beta$ & E.T. & $R^{2}$ ajustado \\
\hline \multirow[t]{6}{*}{ VFP Global } & 1.Agresividad Física & $.58 * * *$ & .05 & .33 \\
\hline & 2.Agresividad Física & $.54 * * *$ & .04 & .46 \\
\hline & FACES Cohesión & $-.36^{*}$ & .04 & \\
\hline & 3.Agresividad Física & $.36^{*}$ & .04 & .55 \\
\hline & FACES Cohesión & $-.36 * *$ & .03 & \\
\hline & CPICS-VER Autoculpa & $-.35^{*}$ & .17 & \\
\hline \multirow[t]{3}{*}{ VFP Física } & 1.FACES Cohesión & $-.48 * *$ & .02 & .23 \\
\hline & 2.FACES Cohesión & $-.46 * *$ & .02 & .4 \\
\hline & CPICS-VER Autoculpa & $-.41 * *$ & .09 & \\
\hline VFP Verbal & 1.Agresividad Física & $.62 * * *$ & .3 & .39 \\
\hline
\end{tabular}

Notas. AQ = Agression Questionnaire (Buss y Perry. 1992); CTS = Conflict Tactics Scales (Straus y Fauchier. 2008); CPIC-VER = The Children's Perception of Interparental Conflict Scale-Versión Española Reducida (Iraurgi et al., 2008); FACES = Family Adaptability and Cohesion Evaluation Scale (Olson. Bell y Portner. 1982); FCS-VE = Family Communication Scale (Barnes y Olson. 1982);VFP = Violencia filio-parental; ${ }^{*} p<.05 ; * * p<.01 ; * * * p<.001$

más, tal y como fue planteado en la primera hipótesis, se halló un número significativamente mayor de estructuras familiares diferentes a la nuclear en el grupo VFP. Así, una mayor proporción de padres separados y familias reconstituidas en el grupo VFP, sugiere que el conflicto conyugal que propició estas estructuras familiares y que puede perdurar más allá de la separación, incrementa situaciones de tensión y/o pérdidas de apoyo familiar (Pagani, Boulerice y Tremblay, 1997).

Los adolescentes inmersos en VFP mostraron mayores niveles de agresividad física e ira. La existencia de diferencias en los niveles de ira, pero no así de hostilidad, podría estar apuntando a que la dimensión emocional de la agresividad tendría un mayor peso que la dimensión cognitiva en la respuesta violenta del adolescente. Estos resultados no coinciden con estudios previos donde se ha hallado una significativa relación entre la VFP y la agresión proactiva (Calvete et al., 2012), la cual podría relacionarse con una mayor hostilidad. En este sentido, el no haber obtenido una mayor hostilidad en VFP y sin embargo sí mayor ira, parece apuntar a que la VFP podría relacionarse en mayor medida con una agresividad de tipo reactivo emocional. Por lo tanto, se hace necesario la profundización en la relación existente entre la dimensión emocional de la agresividad y la violencia proactiva en VFP.

Por otro lado, no se hallaron diferencias significativas entre el GC y VFP en ansiedad, somatización, pánico y niveles de depresión. Al respecto, es conveniente señalar que no es inusual encontrar entre la literatura sobre VFP la hipótesis de que pueda existir una estrecha relación entre la sintomatología ansioso-depresiva de los adolescentes y la expresión de VFP (Calvete et al., 2012; Pereira 2011). Sin embargo, los hallazgos del presente estudio no parecen ir en consonancia con estos resultados. En este sentido, la sintomatología psicológica no hallada en forma de depresión, pánico o ansiedad, puede estar siendo enmascarada a través de la agresividad y la conducta antisocial (Raskin, 1977). Además, no debería descartarse la idea de la deseabilidad social de los adolescentes a la hora de responder como intento de mantener una imagen de invulnerabilidad que puede relacionarse con la grandiosidad y la prepotencia hallada por estudios previos en muestras con análogos clínicos (Aroca-Montolio et al, 2014; Calvete et al., 2011). Por lo tanto, en futuras investigaciones sería adecuado controlar la deseabilidad social mediante una metodología que aunase la utilización de cuestionarios estandarizados con la observación clínica de profesionales entrenados a través de protocolos clínicos bien establecidos. Además, podría resultar de interés analizar si determinadas características personales de los adolescentes como los problemas de autocontrol, bajos niveles de frustración y autoestima, podrían relacionarse en mayor medida con la VFP que con la sintomatología clínica o los trastornos psicológicos, si bien en la actualidad ya existen estudios que señalan la importancia de dichas variables (Cottrell y Monk, 2004; Ibabe et al. 2007).

La tercera hipótesis fue apoyada por los datos en su totalidad. Así, el funcionamiento familiar es percibido con una significativa menor cohesión y adaptabilidad en el grupo VFP. Estos resultados informan sobre un débil 
vínculo emocional y una baja implicación familiar en la convivencia diaria entre los miembros del sistema familiar en el que acontece VFP, así como una baja capacidad para poder adaptarse a acontecimientos vitales del ciclo vital familiar. Un tamaño del efecto mayor en cohesión familiar en comparación con la adaptabilidad, aporta información sobre la importancia del bajo grado de vinculación entre los miembros de la familia, más allá de su capacidad de adaptación a situaciones estresantes en el grupo VFP. Esta baja cohesión familiar en VFP corresponde con lo hallado por Jaureguizar et al., (2013), así como con estudios previos centrados de manera general en el comportamiento agresivo en la adolescencia (Hamama y Arazi, 2011). En el mismo sentido, se evidencia un marcado conflicto conyugal entre los padres de los adolescentes pertenecientes al grupo VFP. Si bien es cierto que profesionales en el tratamiento psicoterapéutico con familias con VFP (Llamazares, Vázquez, y Zuñeda, 2013; Pereira, 2011, p. 124) ya habían informado de conflictos significativos en el subsistema conyugal, se desconocía la percepción del mismo por parte de los hijos en comparación con adolescentes que no ejerciesen violencia en el seno familiar. Así, a excepción de la variable amenaza se obtuvieron diferencias significativas en todas las variables estudiadas respecto al conflicto interparental. Los conflictos conyugales de los padres eran percibidos con mayor intensidad, frecuencia y duración, así como con mayores dificultades para su resolución. Además los motivos de los conflictos conyugales se perciben más relacionados con el propio hijo y estos se consideran más culpables respecto a los mismos.

Finalmente, los adolescentes VFP se sienten menos capaces de manejar el conflicto conyugal de sus padres y más implicados en el mismo. El hecho de no haber obtenido diferencias significativas en amenaza, esto es, en el grado en el que los hijos temen una escalada entre los padres que conlleve ser agredido y verse involucrado en la conflictiva es un hallazgo significativo. En este sentido, el rol adquirido de agresor en la familia junto con lo hallado por previas investigaciones respecto a una significativa creencia de grandiosidad y prepotencia (Aroca-Montolio et al, 2014; Calvete et al., 2011) y combinado con una mayor agresividad física e ira encontrada en el presente estudio, permite al adolescente distanciarse del miedo de que el intenso conflicto conyugal de sus padres pueda dañarle. Respecto a la comunicación familiar, tal y como era de esperar, ante una significativa menor cohesión y adaptabilidad familiar así como un significativo mayor conflicto interparental en VFP comparada con el GC, las habilidades positivas de comunicación familiar como mensajes claros y congruentes, empatía, comunicación de apoyo y habilidades efectivas de reso- lución de problemas, son percibidas significativamente por debajo entre los adolescentes que ejercen violencia en el seno familiar. De esta manera, en la presente casuística se replica lo hallado por previos estudios respecto a la estrecha relación entre el comportamiento violento en la adolescencia y la comunicación negativa entre padres e hijos (Dekovic et al., 2004).

En cuanto a la cuarta hipótesis ésta fue confirmada parcialmente debido a que los resultados no apoyan que una edad de inicio más temprana de la VFP así como una mayor duración de la violencia sin tratamiento se asocie con una mayor gravedad de la misma. En este sentido, la muestra objeto de estudio comenzó a usar la violencia hacia sus progenitores a los 13.9 años, dato similar a lo aportado por previas investigaciones en las que se habla de un rango de edad entre los 10 y los 15 años (Aroca-Montolío et al., 2014). El hecho de que no se haya encontrado una asociación significativa entre la VFP y la edad de inicio o la edad al solicitar tratamiento puede ser debido al amplio rango de edad de la muestra objeto de estudio. Este hallazgo aporta información relevante como para afirmar que no es la edad del adolescente una variable relevante asociada a la VFP y a su gravedad, sino otra serie de variables que serán objeto de análisis más adelante. Igualmente, respecto a la falta de asociación entre el tiempo transcurrido sin tratamiento y la gravedad de la VFP, podría deberse a un fenómeno de efecto techo, a través del cual, una vez que se instaura una dinámica de VFP en el seno de la familia, está no aumenta en gravedad con el paso del tiempo, sino que permanece estática.

En el presente estudio, los resultados apoyan una asociación significativa de la VFP con factores ontogenéticos, como una mayor tendencia hacia la agresividad física, introducida por parte del adolescente en el sistema familiar y factores microsistémicos, como una menor cohesión familiar junto a un alto sentido de autoculpa por parte del adolescente en relación al conflicto conyugal de sus padres. Así, el acontecer de una dinámica de VFP no se asociaría con la dimensión emocional de la agresividad, sino con su aspecto más conductual o con la tendencia al uso de la fuerza física por parte del adolescente. Igualmente, junto a lo apuntado por Pagani et al. (2004), una débil implicación entre padres e hijos en actividades compartidas e interacciones positivas, esto es, una baja cohesión familiar, sería la variable relacionada con el funcionamiento familiar asociada a la VFP global en lugar de la baja adaptabilidad familiar observada. Además, respecto al conflicto interparental, en lugar de la dimensión vivencia amenazante donde queda englobada la triangulación, es la autoculpabilidad la dimensión que se asocia a la VFP. Este resultado con- 
tradice el peso que algunos autores han otorgado a la triangulación como un factor determinante para el surgimiento de la VFP a través del estudio de casos (Micucci, 1995; Pereira y Bertino, 2009). En este sentido, a pesar de que los hijos se impliquen en el conflicto de los padres tomando partido por uno u otro, los resultados apoyan la hipótesis de que es el hecho de que se sienta culpable, uno de los factores determinantes asociados a la VFP. Estos resultados concuerdan con hallazgos obtenidos en investigaciones previas sobre el conflicto interparental y la adaptación de los hijos, ya que variables como la autoculpabilidad o la triangulación son las más relevantes en su relación con el malestar experimentado por los hijos (Iraurgi et al., 2011). Por otro lado, llevando a cabo un análisis diferencial entre la VFP física y la VFP verbal, los resultados mostraron que los factores predictivos de cada forma de VFP eran diferentes. Así, para la VFP física, los únicos factores predictivos eran los microsistémicos, esto es, una baja cohesión familiar y una marcada autoculpabilidad respecto al conflicto interparental. Sin embargo, el único factor predictivo para la VFP verbal fue la agresividad física, entendida esta como una variable ontogenética. De acuerdo a estos resultados, y teniendo en cuenta un modelo en escalada (Walsh y Krienert, 2009), el cual postula que los episodios de violencia comenzarían mediante el uso de una violencia verbal hasta llegar a la instauración de una violencia física, la VFP verbal podría ser explicada desde la agresividad física intraindividual del adolescente. Sin embargo, para llegar a ejercer una VFP física sería necesario una baja cohesión familiar y un significativo conflicto interparental percibido por el adolescente que es atribuido además a sí mismo.

En cuanto a las limitaciones de la presente investigación, en primer lugar se destacan las derivadas del carácter transversal del estudio, el cual no posibilita el establecimiento de relaciones de causalidad entre las diferentes variables ontogenéticas y microsistémicas y la VFP. Otra de las limitaciones se relaciona con el empleo de un muestreo intencional el cual no permite generalizar los resultados a la población adolescente. Sin embargo, si es generalizable a la población clínica objeto de tratamiento de la VFP. Igualmente, debido al tamaño de la muestra no se pudo comprobar las diferencias existentes en función del sexo o de la estructura familiar en las variables estudiadas. Finalmente, los resultados obtenidos en torno a la ausencia de sintomatología ansioso-depresiva no concuerdan con lo hallado por previas investigaciones (Aroca-Montolio et al., 2014; Calvete et al., 2012; Ibabe y Jaureguizar, 2010; Kennedy et al., 2010), quizás por la deseabilidad en las respuestas de los participantes.

A pesar de las limitaciones expuestas, los hallazgos presentados a través de la evaluación de una muestra clínica previa a su inclusión en un tratamiento familiar, aporta datos que tienen una implicación directa para la práctica clínica. En este sentido, mediante la metodología utilizada se logra evitar la dificultad propia de los estudios con análogos clínicos para generalizar los resultados y orientar el tratamiento de la VFP. En suma, los principales resultados sugieren que la VFP se asienta en variables ontogenéticas propias del adolescente como una alta tendencia hacia la agresividad física y variables microsistémicas relacionadas con el funcionamiento familiar, como una baja cohesión entre los miembros de la familia y una alta conflictiva interparental, de la que el adolescente se siente culpable. En este sentido, los resultados orientan hacia la necesidad de un tratamiento que haga hincapié en factores intraindividuales del propio adolescente y en estrategias terapéuticas a implementar con el sistema familiar en su conjunto. Contrastar los resultados obtenidos incluyendo a los padres de los adolescentes implicados en la VFP e implementar estudios que profundicen en el tratamiento de dichas variables, son investigaciones prioritarias de cara al futuro.

\section{Referencias}

Andreu, Y., Galdón, M. J., Dura, E., Ferrando, M., Murgui, S., García, A., \& Ibáñez, E. (2008). Psychometric properties of the Brief Symptoms Inventory-18 (BSI-18) in a Spanish sample of outpatients with psychiatric disorders. Psicothema, 20(4), 844-850.

Aroca-Montolío, C., Lorenzo-Moledo, M., y Miró-Pérez, C. (2014). La violencia filio-parental: Un análisis de sus claves. Anales de Psicología, 30, 157-170.

Arsenio, W., Cooperman, S. y Lover, A. (2000). Affective predictors of preschoolers aggression and peer acceptance: direct and indirect effects. Developmental Psychology, 36, 438-448.

Ballesteros de Valderrama, B.P. (1995). Modelos explicativos de la relación entre conflicto marital y ajuste infantil. Suma psicológica, 2(1), 23-41.

Barnes, H.L., \& Olson, D.H. (1982). Parent-adolescent comunication scale. En Olson, D.H., McCubbin, H.L., Barnes, H.L., Larsen A.S., Muxen, M.J. y Wilson M.C., (Eds.), Family inventaries: Inventaries used in a national survey of families across the family life cycle (pp. 51-63). St. Paul, MN: University of Minnesota.

Berkowitz, L. (1993). Aggression: Its causes, consequences and control. Philadelphia: Temple University Press.

Buss, A.H., y Perry, M.P. (1992). The aggression questionnaire. Journal of Personality and Social Psychology, 63, 452-459.

Calvete, E., y Orue, I. (2011). The impact of violence exposure on aggressive behavior through social information processing in adolescents. American Journal of Orthopsychiatry, 81, 38-50.

Calvete, E., Orue, I., y Gámez-Guadix, M. (2012). Child to parent 
violence: Emotional and behavioral predictors. Journal of Interpersonal Violence, 28, 754-771.

Calvete, E., Orue, I., y Sampedro, R. (2011). Violencia filio-parental en la adolescencia. Características ambientales y personales. Infancia y Aprendizaje: Journal for the Study of Education and Development, 34(3), 349-363.

Cantón, J., Cortés, M. R., y Justicia, M. D. (2007). Conflictos entre los padres, divorcio y desarrollo de los hijos. Madrid: Pirámide.

Cohen, J. (1992). A power primer. Psychological Bulletin, 112(1), 155-159.

Contreras, L., y Cano, M. C. (2015). Exploring psychological features in adolescents who assault their parents: a different profile of young offenders? The Journal of Forensic Psychiatry \& Psychology, 26(2), 224-241.

Cottrell, B. (2001). Parent abuse: the abuse of parents by their teenage children. Ottawa: Health Canada, Family Violence Prevention Unit.

Cottrell, B., y Monk, P. (2004). Adolescent-to-parent abuse. A qualitative overview of common themes. Journal of family issues, 25(8), 1072-1095.

Cummings, E. M., Goeke-Morey, M. C., y Papp L. M. (2004). Everyday marital conflcit and child aggression. Journal of $a b-$ normal child psychology, 32, 191-202.

Dekovic, M., Wissink, I., y Meijer, A. (2004). The role of family and peer relations in adolescent antisocial behaviour: Comparison of four ethnic groups. Journal of Adolescence, 27, 497-514.

Derogatis, L. R. (2001). Brief Symptom Inventory (BSI)-18. Administration, scoring and procedures manual. Minneapolis: NCS Pearson, Inc.

El-Sheikh, M., Buckhalt, J. A., Mize. J., y Acebo, C. (2006). Marital conflict and disruption of children's sleep. Child development, 77. 31-43.

Estévez, E., Murgui, S., Moreno, D., y Musitu, G. (2007). Estilos de comunicación familiar, actitud hacia la autoridad institucional y conducta violenta del adolescente en la escuela. Psicothema, 19, 108-113.

Evans, E. D., y Warren-Sohlberg, L. (1988). A pattern analysis of adolescent abusive behaviour towards parents. Journal of Adolescent Research, 2, 201-216.

Field, A. (2005). Discovering statistics using SPSS (Second Edition). London: Sage Publications.

Gámez-Guadix, M., y Calvete, E. (2012). Violencia filioparental y su asociación con la exposición a la violencia marital y la agresión de padres a hijos. Psicothema, 24, 277-228.

Grych, J. H., Seid, M., y Fincham, F.D. (1992). Assessing marital conflict from the child's perspective. The children's perception of interparental conflict scale. University of Illinois Urbana-Champaign, 63(3), 558-572.

Hamama, L., y Arazi, Y. (2011). Aggressive behaviour in at-risk children: contribution of subjective well-being and family cohesion. Child and Family Social Work, 17, 284-295

Henggeler, S. W. (1989). Delinquency in adolescence. Developmental clinical psychology and psychiatry. Thousand Oaks (USA): Sage.

Ibabe, I., y Jauregizar, J. (2010). Child-to-parent violence: Profile of abusive adolescents and their families. Journal of Criminal Justice, 38, 616-624.

Ibabe, I., Jaureguizar, J., y Bentler, P. M. (2013a). Protective fac- tors for adolescent violence against authority. The Spanish journal of psychology, 16, E76.

Ibabe, I., Jaureguizar, J., y Bentler, P. M. (2013b). Risk factors for child-to-parent violence. Journal of family violence, $28,523-$ 534.

Ibabe, I., Jaureguizar, J., y Díaz, O. (2007). Violencia filio-parental. Conductas violentas de jóvenes hacia sus padres. Vitoria-Gasteiz: Servicio Central de Publicaciones del Gobierno Vasco.

Iraurgi, J., Martínez-Pampliega, A., Iriarte, L., y Sanz, M. (2011). Modelo contextual del conflicto interparental y la adaptación de los hijos. Anales de Psicología, 27(2), 562-573.

Iraurgi, I., Martínez-Pampliega, A., Sanz, M., Cosgaya, L., Galíndez, E., y Muñoz, A. (2008). Escala de conflicto interparental desde la perspectiva de los hijos (Children's Perception of Interparental Conflict Scale - CPIC): Estudio de validación de una versión abreviada de 36 ítems. Revista Iberoamericana de Diagnóstico y Evaluación Psicológica, 25, 9-34.

Jackson, D. (2003). Broadening constructions of family violence: Mothers`s perspectives of aggression from their children. Child and Family Social Work, 8, 321-329.

Jaureguizar, J., Ibabe, I., y Straus, M. A. (2013). Violent and prosocial behavior by adolescents toward parents and teachers in a community sample. Psychology in the Schools, 50(5), 451-470.

Kennedy, T.D., Edmonds, W.A., Dann, K.T., y Burnett, K.F. (2010). The clinical and adaptative features of young offenders with histories of child-parent violence. Journal of family violence, 25, 509-520.

Llamazares, A., Vázquez, G., y Zuñeda, A. (2013). Violencia filio-parental: Propuesta de explicación desde un modelo procesual, Boletín de Psicología, 109, 85-99.

Martínez-Pampliega, A. (2008). Escala de Conflicto Interparental desde la Perspectiva de las/os Hijas/os (CPICS. The Children's Perception of Interpersonal Conflict Scale). En Equipo EIF, Manual de instrumentos de evaluación familiar (pp. 143152). Madrid: CCS.

Martinez-Pampliega, A., Iraurgi, I., Galíndez, E., y Sanz, M. (2006). Family Adaptability and Cohesión Evaluation Scale (FACES): Desarrollo de una versión de 20 ítems en español. International Journal of Clinical and Health Psychology. Revista Intenacional de Psicología Clínica y de la Salud, 6(2), 317-338.

Micucci, J. A. (1995). Adolescents who assault their parents: A family systems approach to treatment. Psychotherapy, 32, 154-161.

Morales-Vives, F., Codorniú-Raga, M. J., y Vigil-Colet, A. (2005). Características psicométricas de las versiones reducidas del cuestionario de agresividad de Buss y Perry. Psicothema, 17(1), 96-100.

Musitu, G., Estévez, E., y Emler, N. (2007). Adjustment problems in the family and school contexts, attitude towards authority and violent behaviour at school in adolescents. Adolescence, 42, 779-794

Nowakowski, E., y Mattern, K. (2014). An Exploratory Study of the Characteristics that Prevent Youth from Completing a Family Violence Diversion Program. Journal of Family Violence, 29(2), 143-149.

Olson, D. H., Bell, R., y Portner, J. (1982). FACES II. Minnesota: Family Social Science.

Olson, D. H., y Gorall, D. M. (2003). Circumplex model of marital 
and family systems. En F. Walhs (Ed.), Normal Family Processes (3 ${ }^{\text {rd }}$ edition) (pp.514-547). New York: Guilford.

Olson, D. H., Sprenkle, D., y Russell, C. (1979). Circumplex model of marital and family systems I: cohesion and adaptability dimensions, family types and clinical applications. Family Process, 18, 3-28.

Pagani, L. S., Boulerice, B., y Tremblay, R. E. (1997). The influence of poverty on children's classroom placement and behaviour problems during elementary school: A change model approach. En G. J. Duncan y J. Brooks-Gun (Eds.), Consequences of growing unpoor (pp. 311-339). New York: Sage.

Pagani, L.S., Tremblay, R.E., Nagin, D., Zoccolillo, M., Vitaro, F., y McDuff, P. (2004). Risk factor models for adolescent verbal and physical aggression toward mothers. International Journal of Behavioral Development, 28(6), 528-537.

Pagani, L.S., Tremblay, R.E., Nagin, D., Zoccolillo, M., Vitaro, F., y McDuff, P. (2009). Risk factor models for adolescent verbal and physical aggression toward fathers. International Journal of Behavioral Development, 24(3), 173-182.

Pereira, R. (2011). Psicoterapia de la violencia filio-parental. Entre el secreto y la vergüenza. Madrid: Morata.

Pereira, R., y Bertino, L. (2009). Una comprensión ecológica de la violencia filio-parental. Redes, Revista de Psicoterapia Relacional e Intervenciones Sociales, 21, 69-90.
Raskin, A. (1977). Depression in children: fact o fallacy. En Schulterbrandt, J. G. y Raskin, A. (Eds.), Depression in childhood: Diagnosis, treatment and conceptual models (pp. 6980). New York, NY: Raven Press.

Rechea, C., Fernández E., y Cuervo A.L. (2008). Menores agresores en el ámbito familiar. Centro de Investigación en criminología, 15, 1-80.

Straus, M., y Fauchier, A. (2008). The International Parenting Study. Recuperado de: http://pubpages.unh.edu/ mas2/IPS. htm

Straus, M. A., Hamby, S. L., Finkelhor, D., Moore, D. W., y Runyan, D. (1998). Identification of child maltreatment with the parent-child conflict tactics scales: Development and psychometric data for a national sample of American parents. Child Abuse and Neglect, 22, 249-270.

Vigil-Colet, A., Lorenzo-Seva, U., Codorniu-Raga, M. J., y Morales, F. (2005). Factor structure of the Buss-Perry aggression questionnaire in different samples and languages. Aggressive Behaviour, 31(6), 601-608.

Walsh, J. A., y Krienert, J. L., (2009). A decade of child-initiated family violence: comparative analysis of child parent violence and parricide examining ofender, victim and event characteristics in a national simple of reported incidents, 1995-2005. Journal of interpersonal violence, 24(9), 1450-1477. 
